Kong. Res. J. 2(2) : 40-54, 2015

ISSN 2349-2694

Kongunadu Arts and Science College, Coimbatore.

\title{
ASSESSMENT OF ECOLOGICAL STATUS OF ECONOMICALLY IMPORTANT PLANTS IN UDHAYAGIRI HILLS, NAGARCOIL
}

\author{
Jenil Prabha, K*. and R. Asir Selin Kumar \\ Postgraduate and Research Department of Botany \\ Scott Christian College (Autonomous), Nagercoil-629 003, Tamil Nadu, India \\ *E.mail: jenilnagercoil@gmail.com
}

\begin{abstract}
\end{abstract}
The present ecological investigation was carried out in the understorey of tropical moist deciduous forest of Udhayagiri fort hills, Kanyakumari, Tamil Nadu. A total number of 171 species were documented in the understory of the study area and among them 163 species were recognized as economically important. The secured value of ecological attribute, importance value index (IVI) indicates that the species like Tephrosia purpurea, Clerodendron infortunatum Acalypa indica, Aerva lanata, Asystasia gangetica, Belpharis maderaptensis, Cleome viscosa, Dodonia viscose, Glycosmis pentaphylla, Leucas aspera, Limnophila indica, Martynia annua, Oldenlandia umbellate, Pavetta indica, Phyllanthus amarus and Rhynchosia minima are well established in the study area. On the other hand, 55 plant species are considered to be ecologically weaker in the community. Hence priorities must be given to these species for natural regeneration and hence their conservation as well.

Keywords: Ecological status, Udhayagiri hills, Importance value index.

\section{INTRODUCTION}

Western Ghats is among the ecologically richest of India, next to the Himalaya in the diversity of its biological species (Gadgil, 1984). It encompasses many types of ecosystems such as tropical wet evergreen forests, dry deciduous forests, moist deciduous forests, thorny scrub jungles and the fragile montane shoals with associated grasslands (Champion and Seth, 1968). Among the different types of vegetations in the Western Ghats, The present study area, Udhayagiri Hills is dominated by the tropical moist deciduous forests. A large number of herbs, shrubs and climbers is commonly occupying in the under storey of the study area. Udhayagiri Hills under semi - arid climatic condition in many parts hold more number of economically and medicinally important plants due to the presence of diverse secondary metabolites. However, works on phytosociological analysis in the understory of this region have been limited. Hence, the present ecological investigation was carried out to enlist economically important plants and to assess their ecological position through phytosociological analysis in the understory of the study area.

\section{MATERIALS AND METHODS}

\subsection{Study area}

The present study area, Udhayagiri fort hills is situated in the Kanyakumari district of Tamil Nadu and lies at a distance of $14 \mathrm{~km}$ from the town of Nagercoil. The Udhayagiri covers a huge area of 22.50 hectares which is surrounded by isolated hills. The elevation of the study area is $97 \mathrm{~m}$ above msl. The geographical location of Udhayagiri fort hills lies between $8^{\circ}-14^{\prime} 38.4 \mathrm{~N}$ attitude and $77^{\circ}-19^{\prime} 55.2 \mathrm{E}$ longitude.

\subsection{Experimental methods}

Phytosociological analysis was carried out during the rainy month of September, 2013 in the understory of the study forest, Udhayagiri hills. At the time of sampling, 50 random quadrats each with the size of $1 \mathrm{~m}^{2}$ were laid to encount the species and their individuals. The quantitative characters such as frequency, density, abundance, relative frequency, relative density, relative dominance and importance value index were calculated according to the following formulae proposed by Cottam and Curtis (1956):

$$
\begin{aligned}
& \text { Frequency }(\%)=\frac{\text { Number of quadrats in which the species present }}{\text { Total number of quadrats studied }} \times 100 \\
& \text { Density }=\frac{\text { Total number of individuals of the species in all quadrats }}{\text { Total number of quadrats studied }} \\
& \text { Abundance }=\frac{\text { Total number of individuals of the species in all quadrats }}{\text { Number of quadrats of occurrence of the species }}
\end{aligned}
$$

Since most of the stems are cylindrical, the basal area was calculated by using the formula:

$$
\text { Basal area } \quad=\pi r^{2}
$$


Where, $\pi=3.14$ and ' $r$ ' is the radius of the stem at the point of emergence.

Relative frequency, relative density, and relative dominance were calculated from the following formulae:

$$
\begin{aligned}
& \text { Relative frequency }(\%)=\frac{\text { Number of occurence of the species }}{\text { Number of occurence of all species }} \times 100 \\
& \text { Relative density }(\%)=\frac{\text { Number of individuals of the species }}{\text { Number of individuals of all species }} \times 100 \\
& \text { Relative dominance }(\%)=\frac{\text { Total basal area of the species }}{\text { Total basal area of all species }} \times 100
\end{aligned}
$$

Importance value Index (IVI) is the sum of quantities of relative frequency, relative density and relative dominance expressed per 300 .

\section{RESULTS AND DISCUSSION}

A total number of 171 species were documented in the understory of the study area and among them 163 were recognized as economically important (Table 1). This may be explained that the study area has favourable microclimate for better growth and development of more number of species and also potential habitat for the plants of economic importance. Puri et at (1989) stated that the continuous availability of moisture in the soils of shoals in Western Ghats enables the appearance of more number species. Despite the presence of suitable microclimate, the constituent species in the understory of shoals in Western Ghats showed wide variation in distribution level, population size and basal area between them (Padmavathy, 2005).

In the present study, the ecological position for highly and poorly established plants alone is highlighted in terms of expressing their frequency, density, basal area and importance value index (Table 2). The dicot species such as Acalypa indica, Asystasia gangetica, Cleome viscose, Leucas aspera, Phyllanthus amarus and Sida cardata showed maximum frequency value of $100 \%$ in the community and certain other herbaceous species viz., Aerva lanata, Belpharis maderaptensis, Mollugo pentaphylla, Oldenlandia umbellate, Stylosantus hamate and Tephrosia purpurea also showed higher distribution (around 85\% frequency value) during rainy month of September. The higher seed output and greater reproductive potential exist in these species may be the possible reasons for this fact (Usher, 1991).

Many species in the understory of the study area like Amaranthus spinnosus, Amaranthus virigidis, Barleria buxifolia, Biophytum sensitivum,
Croton zeylanicus, Crotalaria pallida, Curculigo orchioides, Plumbago zeylanica and Rauvolfia serpentina have exhibited poor distribution. The external factors like topography, soil conditions and the biotic disturbance and some intrinsic factors like dispersal mechanism, longevity of seeds, duration of dormancy and germination efficiency are some of the environmental variables generally determine the degree of distribution of any plant species (Belsky, 1988).

The plant species such as Tephrosia purpurea, Acalypa indica, Aerva lanata, Asystasia gangetica, Belpharis maderaptensis, Cleome viscosa, Clerodendron infortunatum, Leucas aspera,

Phyllanthus amarus, Sida cardata, Mollugo pentaphylla and Oldenlandia umbellata were present in the study area with higher densities. This may be due to the presence of continuous wetness, a favourable factor for the better growth of these species in the soil of moist deciduous forest (Saxena, 1991). On the other hand, many species like Rauvolfia serpentina, Cardiospermum halicacabum and Borreria articularis, Andrographis paniculata, Boerhavia diffusa, Blainviella acmella, Cleome rutidesperma, Cleome rutidesperma, Croton zeylanicus, Desmodium illinoensis, Rungia repens, Sida cordifolia, Barleria cuspidata, Aerva lanata, Amaranthus virgatus, Clitoria ternate, Corchorus aestuans and Croton bonplandiam, Belpharis maderaptensis, Gymnema sylvestre, Heydyotis peterita, Ocimum americanum, Ludwigia octovalvis etc., have always present with low densities in the study area. The poor reproductive potential with less seed output and weaker competitive ability may lead the species with low density in the communities (Chandrasakaran and Swamy, 1995).

The basal cover of certain plant species such as Tephrosia purpurea, Clerodendron infortunatum, Acalypa indica and Pavetta indica was greater in the understory of studied forest. Among them, due to shrubby habit two species namely Clerodendron infortunatum and Pavetta indica were occupied higher basal cover in comparison to other constituent species. This feature may lead to the occupation of higher basal cover in the communities. The lower basal cover attained by many species such as Aerva javanica, Alternanthera sessilis, Asparagus racemosus, Centella asiatica, Clitoria ternate, Desmodium adscendens, D. triflorum, Evolvulus alsinoides, E. nummularis, Indigofera hirsuta, I. glandulosa, Justicia gluca, and Oldenlandia corymbosa might be due to their poor ecological characters like lower density and less basal area per individual. 
Table 1. The constituent species in the study area, Udhayagiri hills with their ecological status and economic importance.

\begin{tabular}{|c|c|c|c|c|c|}
\hline $\begin{array}{l}\text { S. } \\
\text { No. }\end{array}$ & Species & $\begin{array}{c}\text { Ecological } \\
\text { status }\end{array}$ & Parts used & Medicinal/Other economic importance & $\begin{array}{c}\text { Mode of } \\
\text { administration }\end{array}$ \\
\hline 1 & Abrus pulchellus & Common & Leaves & Cure fever, cough, cold & Juice \\
\hline 2 & Abulition indicum (L.). Sw & Common & Root & Piles & Extract \\
\hline 3 & Acalypha indica L. & Common & Leaves & Headache, wounds, itching & Juice, paste \\
\hline 4 & Acanthospermum hispidum DC. & Common & Root & Jaundice & Decoction \\
\hline 5 & Achyranthus aspera Linn. & Common & spike & Poisonous insect bites. & Paste \\
\hline 6 & Aerva javanica & Common & Whole plant & Swelling & Decoction \\
\hline 7 & Aerva lanata (L.) Juss.ex.Shut. & Common & Root & Piles & Paste \\
\hline 8 & Alternanthera pungens Kunth. & Common & Whole plant & Gonorrhea & Decoction \\
\hline 9 & Alternanthera tenella & Common & Inflorescences & Earache & Ash \\
\hline 10 & Amaranthus spinosus L. & Common & Whole plant & Thorns & Paste \\
\hline 11 & Amaranthus viridis Linn. & Common & Leaves, root & $\begin{array}{l}\text { Scorpion sting, diuretic, laxative, retention of } \\
\text { urine, treat gonorrhea }\end{array}$ & Decoction, paste \\
\hline 12 & $\begin{array}{l}\text { Andrographis echioides (Burm.f.) } \\
\text { Nees }\end{array}$ & Common & Leaf & Head ache & Paste \\
\hline 13 & $\begin{array}{l}\text { Andrographis paniculata (Burn.F) } \\
\text { Wall.ex.Nees. }\end{array}$ & Common & Root, leaves & Fever, liver complaints and jaundice & Decoction, paste \\
\hline 14 & Asparagus racemosus Willd.(L.) & Common & Root tubers & Kidney stone, week end immunity & Powder, paste \\
\hline 15 & $\begin{array}{l}\text { Asystasia gangetica. (L.) T. } \\
\text { Anderson in Thwaittes. }\end{array}$ & Common & Whole plant & Rheumatism & Juice \\
\hline 16 & Barleria buxifolia (L.) & Common & Leaves & $\begin{array}{l}\text { Viral fever, urinary affection, stomach } \\
\text { disorders }\end{array}$ & Juice \\
\hline 17 & Barleria cuspidate & Common & Leaves, flower & Viral fever & Decoction \\
\hline 18 & Barleria lupulina & Rare, endemic & Leaves & Urinary tract infection & Juice \\
\hline 19 & $\begin{array}{l}\text { Belpharis maderaptensis (L.) B. } \\
\text { Heyne. Ex. Roth. Nov. }\end{array}$ & Common & Leaves & Bone fracture & Paste \\
\hline 20 & Biophytum sensitivum & Common & Leaves, roots & Bite poisoning, wound & Paste \\
\hline 21 & Blainviella acmella & Common & Leaves & Alcohol deaddiction & Juice \\
\hline 22 & Boerhavia diffusa L. & Common & Root & Asthma, sugar in urine & Decoction, juice \\
\hline 23 & Borreria latifolia & Common & Leaves, stem & Body pain & Crushed \\
\hline 24 & $\begin{array}{l}\text { Bryophyllum pinnatum (lam) } \\
\text { oken. Alleg }\end{array}$ & Common & Leaves & Dysentery cuts and wounds, head ache. & Juice, paste \\
\hline 25 & Cardiospermum halicacabum L. & Common & Leaves & Cough piles, arthritis, joint pains, skin diseases & Decoction \\
\hline 27 & Celosia argentea $\mathrm{L}$. & Common & Whole plant & Urinary stones & Decoction \\
\hline
\end{tabular}


30 Chromolaena oderata (L.) King \& Robi

31 Cleome aspera

Cleome gynandra $\mathrm{L}$.

Cleome monophylla

Cleome rutidesperma

Cleome viscosa $\mathrm{L}$

Clerodendram infortunatum auct. Non L.

$37 \quad$ Clitoria ternata L.

Commelina benghalensis Linn.

Corchorus aestuans L.Syst. Nat

Corchorus olitorius L.

Crotalaria mysorensis Roth.

Crotalaria pallid

Crotalaria verrucosa $\mathrm{L}$.

\section{Croton bonplandiam Bail}

Croton hirtus

Croton zeylanicus

Cuphea hyrsopifolia

Curculigo orchioides Gaertn.

Cyanotis axilaris

Cyanotis tuberose

Cynodon doctylon (L.) Pers

Panicum Dactylon Linn.

52 Cyperus rotundus L.

Cyrtococcum patens

Datura metal L.

Desmodium adscendens

Desmodium illinoensis

Desmodium triflorum (Linn.) Dc.

Dodonaea viscosa (L.) Jacq

\begin{tabular}{|c|c|c|}
\hline Common & Whole plant & Typhoid \\
\hline Endangered & Root & Head ache, diarrhea, stop vomiting \\
\hline Common & Whole plant & Wounds and rashes \\
\hline Endemic & - & - \\
\hline Common & Leaf & Wounds, fever \\
\hline Endemic & Leaves, root & Vomit \\
\hline Common & Whole plant & $\begin{array}{l}\text { To relief general sickness and uneasiness of } \\
\text { the body }\end{array}$ \\
\hline Common & Leaves & Earache, wound \\
\hline Common & Leaves & $\begin{array}{l}\text { Tumors, hair growth, wounds and fungal } \\
\text { infection. }\end{array}$ \\
\hline Common & Whole plant & Snake bite, cause abortion \\
\hline Common & Whole plant & Leprosy, jaundice \\
\hline Common & Leaves & Head ache \\
\hline Vulnerable & Seed & Stomach ache \\
\hline Common & Leaf, fruit & Stomach ache and stomach ulcer \\
\hline Common & $\begin{array}{l}\text { Seed, leaf, whole } \\
\text { plant }\end{array}$ & Skin diseases, ring worm, itches, stomach pain \\
\hline Common & Leaves & Skin allergies \\
\hline Rare, endemic & Latex & Bleeding and venereal sores, headache \\
\hline Common & - & - \\
\hline Common & Bark & Stomachache \\
\hline Common & Leaves & Cold \\
\hline Common & Root & $\begin{array}{l}\text { Ulcer, treat asthma, piles, jaundice, diarrhea, } \\
\text { and gonorrhea }\end{array}$ \\
\hline Common & Whole plant & Rheumatism, joint pain \\
\hline Common & Tubers & Relief cough \\
\hline Common & Whole plant & Diuretic \\
\hline Common & Tuber & Stomach ache \\
\hline Common & - & - \\
\hline Common & Leaves & Asthma, chronic ulcer \\
\hline Common & Leaves & Wounds, venereal sores \\
\hline Common & Leaves & Itches \\
\hline Common & Leaves, root & Diarrhea, cough, asthma \\
\hline Common & Leaves & Tooth ache \\
\hline
\end{tabular}

Juice

Powder

Paste

-

Paste, decoction

Powder

Infusion

Juice, paste

Paste

Extract

Paste, juice

Poultice

Powder

Paste

Paste, decoction

Extract

Juice

uice

Infusion

Juice

Decoction

Eaten

Extract

Paste

Powder

Boiled

Paste, juice

Juice 


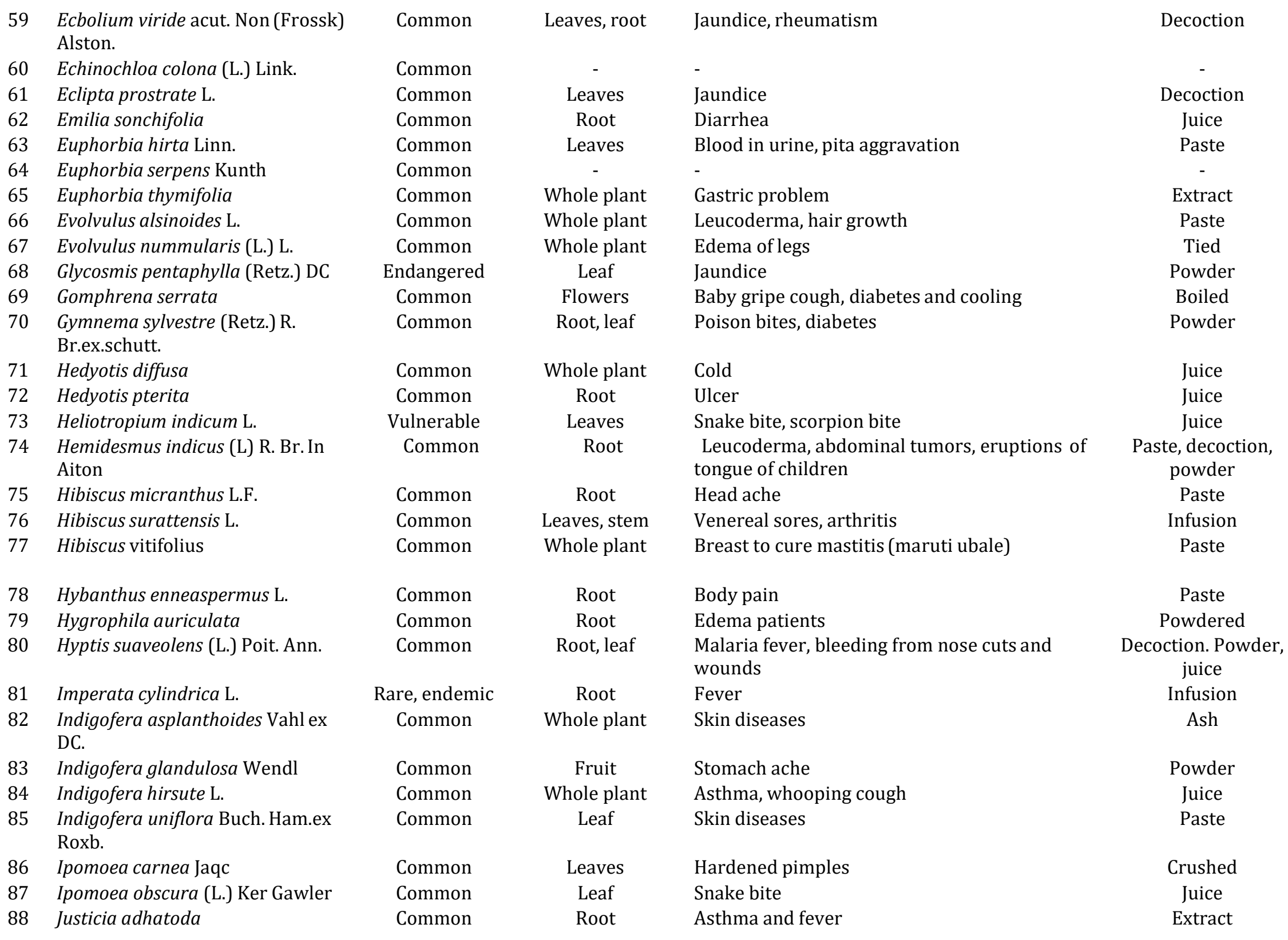




\section{Common}

Kyllinga odorata

Lagascea mollis Cav

Common

Common

Lantana camera Linn.

Common

Leucas aspera (Wild). Link, Enum.

Leucas grandis

Linderina crustacea

98 Lindernia ciliate

99 Ludwigia octovalvis (Jacq.) Raven.

100 Ludwigia peruviana

101 Malvastrum coromandelianum (L.)

Garcke

102 Martynia annua

103 Melhania hamiltoiana

104 Melochia corchorifolia L.

105 Merremia tridentate (L.) Hallier

106 Microstachys chamaelea

107 Mimosa pudica L.

108 Mollugo pentaphylla L.

109 Naregamia alata Wight \& Arn

110 Ocimum americanum L.

111 Ocimum basilicum L.

112 Ocimum gratissimum L.

113 Ocimum tenuiflorum

114 Oldenlandia corymbosa $\mathrm{L}$.

115 Oldenlandia umbellate $\mathrm{L}$.

116 Orthosiphon thymiflorus (Roth)

Sleesen, Reinwandtia

117 Osbeckia aspera

118 Pavetta indica $\mathrm{L}$.
Common

Common

Common

Common

Common

Common

Common

Common

Common

Common

Common

Common

Common

Common

Common

Rare, endemic

Common

Endemic

Common

Common

Common

Common

Common

Common

\section{Leaves}

Leaves

Root, leaf

Whole plant

Leaves

Leaf, flower

Flower

Leaves

Leaves, whole plant

Whole plant

Leaves

Leaves

Root, leaves

Leaves

Leaves

Leaf, root

Whole plant

Root

Leaf

Whole plant, root

Leaf

Leaf

Leaves

Whole plant, leaf

Whole plant

Leaf, root

Leaves

Root, leaves
Back ache

Strengthening of bones

Jaundice. Skin aliments

Diarrhea

Chest and throat to cure cold

Measles and chicken pox, malarial fever, ring

Itch scabies, blockage of nose, head ache

Alleviate fever

Ring worm

Ring worm, clear stomach

Fungal infection of toes

Urine problem of children

Wounds and dysentery

Snake bite, epilepsy, tuberculosis

Dysentery, wounds

Stomach disorders

Growth of the hair, diabetes

Head ache

Whylous urine, veterinary

Cooling purpose, urinary troubles

tch and contagious skin diseases and dysentery

Cuts and wounds

Acne vulgarism, pimples, earache, nasal congestion

Cough, fever, nasal catarrh

Leucoderma, common fever, cold and cough

Liver trouble, urinary disorder in children, jaundice, fever and bilious infection.

Asthma, bronchitis

Skin eruption

Urinary diseases, ulcerated nose
Extract, paste

Juice

Juice

Extract

Juice, paste

Poultice

Paste

Decoction, paste

Paste

Paste

Paste

Paste, juice

Paste

Decoction

Decoction

Decoction, juice

Decoction

Decoction

Paste, decoction

Paste

Decoction, paste

Boil, juice

Extract, decoction

Juice

Juice

Infusion

Paste, decoction,

juice

Juice

$-$

Decoction 


\begin{tabular}{|c|c|}
\hline 119 & Pedalium murex L. \\
\hline 120 & Pergularia daemia Forssk. \\
\hline 121 & $\begin{array}{l}\text { Peristrophe paniculate (Forsk). } \\
\text { Burm. }\end{array}$ \\
\hline 122 & Persicaria hydropiper L. \\
\hline 123 & Phryma laptostachya \\
\hline 124 & Phyla nodiflora (L.)Greene. \\
\hline 125 & $\begin{array}{l}\text { Phyllanthus amarus Schumach \& } \\
\text { Thonn. }\end{array}$ \\
\hline 126 & Phyllanthus debilis Klein ex willed. \\
\hline 127 & Phyllanthus maderrasptensis L. \\
\hline 128 & Phyllanthus myrtifolius \\
\hline 129 & Phyllanthus virgatus G.Forest.FI. \\
\hline 130 & Physalis minima $\mathrm{L}$. \\
\hline 131 & Plumbago zeylanica Linn. \\
\hline 132 & Polycarpaea corymbosa (L.) Lam. \\
\hline 133 & polygala chinensis \\
\hline 134 & Portulaca quadrifida \\
\hline 135 & $\begin{array}{l}\text { Psuedarthria viscida (L) Wight\& } \\
\text { Arn. }\end{array}$ \\
\hline 136 & $\begin{array}{l}\text { Rauvolfia serpentine } \\
\text { (Linn.)Benth.ex Kurz }\end{array}$ \\
\hline 137 & $\begin{array}{l}\text { Rhinacanthus nasutus (L.) Kurz. } \\
\text { J.Asiat }\end{array}$ \\
\hline 138 & Rhynchosia minima (L.) DC. \\
\hline 139 & Rulliea prostrate \\
\hline 140 & Rulliea tuberosa L. \\
\hline 141 & Rungia repens (L.) Nees. \\
\hline 142 & Scoparia dulcis Linn. \\
\hline 26 & Senna absus \\
\hline 143 & Senna occidentalis \\
\hline 144 & Senna uniflora \\
\hline 145 & Sida acuta Burm F.FI. \\
\hline 146 & $\begin{array}{l}\text { Sida cordata (Burm.f.) } \\
\text { Borss.Waalk. }\end{array}$ \\
\hline
\end{tabular}

\begin{tabular}{|c|c|c|}
\hline Common & Leaves & Gonorrhea \\
\hline Common & Leaf & Head ache and asthma \\
\hline Common & Fruit & Eye problem \\
\hline Common & Leaves & Cold and cough \\
\hline Common & Root & Skin diseases \\
\hline Endemic & Leaves & Leucorrhoea, dandruff \\
\hline Common & Whole plant, root & Skin diseases, body heat, fever, jaundice \\
\hline Common & Leaves & Sickle cell anemia \\
\hline Common & Fruits & Teeth diseases \\
\hline Common & Root & Jaundice \\
\hline Common & Leaves & Eye diseases \\
\hline Common & Leaf & Stomach to cure boils \\
\hline Common & Roots & $\begin{array}{l}\text { Fever, skin diseases, diuretic, rheumatism and } \\
\text { dyspepsia. }\end{array}$ \\
\hline Common & Leaf & Jaundice \\
\hline Common & Leaves & Stopping mother feeding \\
\hline Common & Leaves & Swellings erysipelas, burns, scalds \\
\hline Common & Root & Asthma, fever, diarrhea, worms, piles \\
\hline Endangered & $\begin{array}{l}\text { Root, rhizome, } \\
\text { leaves }\end{array}$ & Dysentery \\
\hline Endemic & Leaf, root & Snake and skin diseases. \\
\hline Common & Whole plant & Delivery for body care \\
\hline Common & Whole plant & Diabetes \\
\hline Common & Leaves & Asthma, sinking of ribs \\
\hline Endemic & Whole plant & Bronchitis, fever \\
\hline Common & Seed, leaf & Kidney stone, common fever, throat sore \\
\hline Endemic & Leaves, seeds & Cough, ringworm, skin diseases \\
\hline Common & Seed, leaf, root & Skin disease, head bone fractures \\
\hline Common & Leaves & Wounds, cure eczema \\
\hline Common & Leaves & Wounds \\
\hline Common & Roots & Nervous, urinary diseases \\
\hline
\end{tabular}

Juice
Paste
Juice
Infusion
Paste, infusion
Powder, paste
Paste
Juice
Powder
Paste
Juice
Juice
Powder
Paste
Paste
Paste
Juice
Decoction,
powdered
paste
Juice
Infusion
Juice
Decoction
Powder and
decoction
Juice and paste
Paste
Poultice
Paste
Infusion




\begin{tabular}{|c|c|c|c|c|c|}
\hline 147 & Sida cordifolia L. & Common & Root & Refrigerant & Paste \\
\hline 148 & Solanum melongena & Common & Seeds, leaves & $\begin{array}{l}\text { Cold, cough, phlegm accumulation, gum } \\
\text { infection, tooth ache. }\end{array}$ & Powder \\
\hline 149 & Solanum torvum Swartz & Common & Fruit & Anemia, chest congestion cough, cold. & Paste \\
\hline 150 & Solanum virginianum & Common & $\begin{array}{l}\text { Fruits, whole } \\
\text { plant }\end{array}$ & Cough, asthma, tooth ache, chest pain & Decoction, juice \\
\hline 151 & Spermacoce articularis & Common & Whole plant & Head ache & Decoction \\
\hline 152 & Spermacoce hispida & Common & Root & Urinary infection, headache, internal heat & Decoction \\
\hline 153 & Spermacoce ocymoides Burm.F & Common & Leaves & Diarrhea and dysentery & Infusion \\
\hline 154 & Spermacoce pusilla & Common & Roots & Urinary infection, headache, internal heat & Decoction \\
\hline 155 & $\begin{array}{l}\text { Stashytropheta jamaicensis (L.) } \\
\text { Vahl.Enum. }\end{array}$ & Common & Whole plant & Stomach pains & Decoction \\
\hline 156 & Stylosanthus fruticosa & Common & Whole plant & Febrifuge & Infusion \\
\hline 157 & Stylosantus hamata & Common & Whole plant & Diarrhea and cold & Juice \\
\hline 158 & $\begin{array}{l}\text { Synederella nodiflora (L). } \\
\text { Gaertn.Fruct.Sem. }\end{array}$ & Common & Leaves & Itch scabies & Juice \\
\hline 159 & Tephrosia purpurea (L.) Pers. & Common & Root, flower & Dyspepsia, eye inflammation & Decoction, juice \\
\hline 160 & Trianthema portulacastrum Linn & Common & Leaf & Urinary troubles & Juice \\
\hline 161 & Tribulus terrestris Linn & Common & $\begin{array}{l}\text { Fruit, whole } \\
\text { plants }\end{array}$ & Urinary problem, kidney stones, stomach ache & Powder, extract \\
\hline 162 & Trichodesmum indicum (L.) R.Br & Common & Leaf, root & Scabies, swelling of joints & Paste, powder \\
\hline 163 & Tridax procumbens $\mathrm{L}$. & Common & Leaves & Head ache, cuts, wounds & Juice, paste \\
\hline 164 & $\begin{array}{l}\text { Triumfetta rhomboidea Jacq. } \\
\text { Enum. Sy st. }\end{array}$ & Common & Roots & Bone fracture & Paste \\
\hline 165 & Urena lobata Linn. & Common & Root & Body edema & Paste \\
\hline 166 & Urena sinuate & Common & Root & Urinary disease & Decoction \\
\hline 167 & $\begin{array}{l}\text { Vernonia cineriea (L.) } \\
\text { Less.Linnaea. }\end{array}$ & Common & Whole plant & Wounds & Paste \\
\hline 168 & Vigna triblobata (L.)Verde. & Rare & Root & Till the person vomits in snake bite & Powder \\
\hline 169 & Waltheria indicum L. & Vulnerable & Roots, leaves & Washing wounds & Decoction \\
\hline 170 & Xanthium stumarium & Common & Leaves & Infection fingers & Paste \\
\hline 171 & Zorina diphylla (L.) Pers. & Common & Whole plant & Breast to cure mastitis (maruti ubale) & Paste \\
\hline
\end{tabular}


Table 2. Number of individuals in 50 quadrats $(1 \times 1 \mathrm{~m}$ each) and quantitative characters such as frequency, density, abundance, basal area, relative frequency, relative density, relative dominance and IVI of constituent species in the study forest during the rainy month of September 2013.

\begin{tabular}{|c|c|c|c|c|c|c|c|c|c|c|}
\hline $\begin{array}{c}\text { S. } \\
\text { No }\end{array}$ & Species & $\begin{array}{l}2013 \\
\text { SEP }\end{array}$ & $\begin{array}{c}\text { Frequency } \\
\%\end{array}$ & $\begin{array}{c}\text { Density } \\
\text { (indi./m2) }\end{array}$ & Abundance & $\begin{array}{c}\text { Basel area } \\
\text { (sq.mm/50quad.) }\end{array}$ & $\begin{array}{c}\text { Relative } \\
\text { frequency } \\
(\%)\end{array}$ & $\begin{array}{c}\text { Relative } \\
\text { density } \\
(\%)\end{array}$ & $\begin{array}{c}\text { Relative } \\
\text { dominance } \\
(\%)\end{array}$ & IVI \\
\hline 1 & Abrus pulchellus & $42(29)$ & 58 & 0.84 & 1.45 & 2.41 & 0.86 & 0.96 & 0.16 & 1.98 \\
\hline 2 & Abulition indicum & $32(25)$ & 50 & 0.64 & 1.28 & 8.61 & 0.74 & 0.73 & 0.57 & 2.04 \\
\hline 3 & Acalypa indica & $61(50)$ & 100 & 1.22 & 1.22 & 42.84 & 1.49 & 1.39 & 2.81 & 5.69 \\
\hline 4 & $\begin{array}{l}\text { Acanthospermum } \\
\text { hispidum }\end{array}$ & $29(15)$ & 30 & 0.58 & 1.93 & 3.74 & 0.45 & 0.66 & 0.25 & 1.35 \\
\hline 5 & Achyranthus aspera & $40(29)$ & 58 & 0.8 & 1.38 & 4.08 & 0.86 & 0.91 & 0.27 & 2.04 \\
\hline 6 & Aerva javanica & $19(10)$ & 20 & 0.38 & 1.90 & 0.27 & 0.30 & 0.43 & 0.02 & 0.75 \\
\hline 7 & Aerva lanata & $70(49)$ & 98 & 1.4 & 1.43 & 4.01 & 1.46 & 1.60 & 0.26 & 3.32 \\
\hline 8 & $\begin{array}{l}\text { Alternanthera } \\
\text { pungens }\end{array}$ & $20(15)$ & 30 & 0.4 & 1.33 & 2.58 & 0.45 & 0.46 & 0.17 & 1.07 \\
\hline 9 & Alternanthera tenella & $17(15)$ & 30 & 0.34 & 1.13 & 0.68 & 0.45 & 0.39 & 0.04 & 0.88 \\
\hline 10 & $\begin{array}{l}\text { Amaranthus } \\
\text { spinnosus }\end{array}$ & $15(10)$ & 20 & 0.3 & 1.50 & 4.04 & 0.30 & 0.34 & 0.26 & 0.90 \\
\hline 11 & Amaranthus virigidis & $16(12)$ & 24 & 0.32 & 1.33 & 3.08 & 0.36 & 0.36 & 0.20 & 0.92 \\
\hline 12 & $\begin{array}{l}\text { Andrographis } \\
\text { echoides }\end{array}$ & $20(15)$ & 30 & 0.4 & 1.33 & 3.85 & 0.45 & 0.46 & 0.25 & 1.16 \\
\hline 13 & $\begin{array}{l}\text { Andrographis } \\
\text { paniculata }\end{array}$ & $12(9)$ & 18 & 0.24 & 1.33 & 6.19 & 0.27 & 0.27 & 0.41 & 0.95 \\
\hline 14 & Asparagus racemosus & $14(10)$ & 20 & 0.28 & 1.40 & 0.09 & 0.30 & 0.32 & 0.01 & 0.62 \\
\hline 15 & Asystasia gangetica & $62(50)$ & 100 & 1.24 & 1.24 & 2.47 & 1.49 & 1.41 & 0.16 & 3.06 \\
\hline 16 & Barleria cuspidata & $19(13)$ & 22 & 0.32 & 1.45 & 14.68 & 0.33 & 0.36 & 0.96 & 1.66 \\
\hline 17 & Barleria buxifolia & $16(11)$ & 26 & 0.38 & 1.46 & 14.64 & 0.39 & 0.43 & 0.96 & 1.78 \\
\hline 18 & Barleria lupulina & $26(19)$ & 38 & 0.52 & 1.37 & 18.26 & 0.57 & 0.59 & 1.20 & 2.36 \\
\hline 19 & $\begin{array}{l}\text { Belpharis } \\
\text { maderaptensis }\end{array}$ & $69(49)$ & 98 & 1.38 & 1.41 & 5.38 & 1.46 & 1.57 & 0.35 & 3.39 \\
\hline 20 & $\begin{array}{l}\text { Biophytum } \\
\text { sensitivum }\end{array}$ & $9(7)$ & 14 & 0.18 & 1.29 & 2.06 & 0.21 & 0.21 & 0.14 & 0.55 \\
\hline 21 & Blainviella acmella & $15(11)$ & 22 & 0.3 & 1.36 & 7.74 & 0.33 & 0.34 & 0.51 & 1.18 \\
\hline 22 & Boerhavia diffusa & $18(13)$ & 26 & 0.36 & 1.38 & 1.83 & 0.39 & 0.41 & 0.12 & 0.92 \\
\hline 23 & Borreria latifolia & $20(17)$ & 34 & 0.4 & 1.18 & 8.15 & 0.51 & 0.46 & 0.54 & 1.50 \\
\hline
\end{tabular}




\begin{tabular}{|c|c|c|c|c|c|c|c|c|c|c|}
\hline 24 & $\begin{array}{l}\text { Bryophyllum } \\
\text { pinnatum }\end{array}$ & $20(16)$ & 32 & 0.4 & 1.25 & 16.85 & 0.48 & 0.46 & 1.11 & 2.04 \\
\hline 25 & $\begin{array}{l}\text { Cardiospermum } \\
\text { halicabum }\end{array}$ & $15(10)$ & 20 & 0.3 & 1.50 & 3.44 & 0.30 & 0.34 & 0.23 & 0.87 \\
\hline 26 & Cassia absus & $27(25)$ & 50 & 0.54 & 1.08 & 2.11 & 0.74 & 0.62 & 0.14 & 1.50 \\
\hline 27 & Celosia argentea & $18(15)$ & 30 & 0.36 & 1.20 & 5.62 & 0.45 & 0.41 & 0.37 & 1.23 \\
\hline 28 & Centella asiatica & $15(13)$ & 26 & 0.3 & 1.15 & 0.10 & 0.39 & 0.34 & 0.01 & 0.74 \\
\hline 29 & $\begin{array}{l}\text { Cheilocostus } \\
\text { speciosus }\end{array}$ & $16(13)$ & 26 & 0.32 & 1.23 & 14.68 & 0.39 & 0.36 & 0.96 & 1.72 \\
\hline 30 & Chromolaena oderata & $14(12)$ & 24 & 0.28 & 1.17 & 8.05 & 0.36 & 0.32 & 0.53 & 1.20 \\
\hline 31 & Cleome aspera & $20(11)$ & 22 & 0.4 & 1.82 & 4.59 & 0.33 & 0.46 & 0.30 & 1.08 \\
\hline 32 & Cleome gynandra & $25(15)$ & 30 & 0.5 & 1.67 & 14.37 & 0.45 & 0.57 & 0.94 & 1.96 \\
\hline 33 & Cleome monophylla & $19(12)$ & 24 & 0.38 & 1.58 & 5.93 & 0.36 & 0.43 & 0.39 & 1.18 \\
\hline 34 & Cleome rutidesperma & $18(13)$ & 26 & 0.36 & 1.38 & 6.45 & 0.39 & 0.41 & 0.42 & 1.22 \\
\hline 35 & Cleome viscose & $65(50)$ & 100 & 1.3 & 1.30 & 37.36 & 1.49 & 1.48 & 2.45 & 5.42 \\
\hline 36 & $\begin{array}{l}\text { Clerodendran } \\
\text { infortunatum }\end{array}$ & $72(48)$ & 96 & 1.44 & 1.50 & 60.65 & 1.43 & 1.64 & 3.98 & 7.05 \\
\hline 37 & Clitoria ternate & $13(11)$ & 22 & 0.26 & 1.18 & 0.19 & 0.33 & 0.30 & 0.01 & 0.64 \\
\hline 38 & $\begin{array}{l}\text { Commelina } \\
\text { benghalensis }\end{array}$ & $15(10)$ & 20 & 0.3 & 1.50 & 1.93 & 0.30 & 0.34 & 0.13 & 0.77 \\
\hline 39 & Corchorus aestuans & $15(13)$ & 26 & 0.3 & 1.15 & 1.93 & 0.39 & 0.34 & 0.13 & 0.86 \\
\hline 40 & Corchorus olitorius & $25(23)$ & 46 & 0.5 & 1.09 & 10.19 & 0.68 & 0.57 & 0.67 & 1.92 \\
\hline 41 & Crotalaria mysorensis & $12(11)$ & 22 & 0.24 & 1.09 & 10.11 & 0.33 & 0.27 & 0.66 & 1.26 \\
\hline 42 & Crotalaria pallida & $16(12)$ & 24 & 0.32 & 1.33 & 17.22 & 0.36 & 0.36 & 1.13 & 1.85 \\
\hline 43 & Crotalaria verrucosa & $25(19)$ & 38 & 0.5 & 1.32 & 14.37 & 0.57 & 0.57 & 0.94 & 2.08 \\
\hline 44 & $\begin{array}{l}\text { Croton } \\
\text { bonplandianum }\end{array}$ & $12(9)$ & 18 & 0.24 & 1.33 & 5.52 & 0.27 & 0.27 & 0.36 & 0.90 \\
\hline 45 & Croton hirtus & $13(9)$ & 18 & 0.26 & 1.44 & 7.47 & 0.27 & 0.30 & 0.49 & 1.05 \\
\hline 46 & Croton zeylanicus & $9(7)$ & 14 & 0.18 & 1.29 & 4.64 & 0.21 & 0.21 & 0.30 & 0.72 \\
\hline 47 & Cuphea hyrsopifolia & $19(15)$ & 30 & 0.38 & 1.27 & 2.45 & 0.45 & 0.43 & 0.16 & 1.04 \\
\hline 48 & Curculigo orchioides & $10(8)$ & 16 & 0.2 & 1.25 & 7.71 & 0.24 & 0.23 & 0.51 & 0.97 \\
\hline 49 & Cyanotis axillaries & $13(9)$ & 18 & 0.26 & 1.44 & 1.32 & 0.27 & 0.30 & 0.09 & 0.65 \\
\hline 50 & Cyanotis tuberose & $18(14)$ & 28 & 0.36 & 1.29 & 1.40 & 0.42 & 0.41 & 0.09 & 0.92 \\
\hline 51 & Cynodon doctylon & $34(19)$ & 38 & 0.68 & 1.79 & 1.95 & 0.57 & 0.78 & 0.13 & 1.47 \\
\hline 52 & Cyperus rotundus & $35(26)$ & 52 & 0.7 & 1.35 & 2.73 & 0.77 & 0.80 & 0.18 & 1.75 \\
\hline 53 & Cyrtococcum patens & $28(25)$ & 50 & 0.56 & 1.12 & 6.42 & 0.74 & 0.64 & 0.42 & 1.80 \\
\hline 54 & Datura metal & $19(12)$ & 24 & 0.38 & 1.58 & 18.91 & 0.36 & 0.43 & 1.24 & 2.03 \\
\hline
\end{tabular}




\begin{tabular}{|c|c|c|c|c|c|c|c|c|c|c|}
\hline 55 & $\begin{array}{l}\text { Desmodium } \\
\text { adscendens }\end{array}$ & 19(17) & 34 & 0.38 & 1.12 & 0.27 & 0.51 & 0.43 & 0.02 & 0.96 \\
\hline 56 & $\begin{array}{l}\text { Desmodium } \\
\text { illinoensis }\end{array}$ & $15(12)$ & 24 & 0.3 & 1.25 & 4.04 & 0.36 & 0.34 & 0.26 & 0.96 \\
\hline 57 & Desmodium triflorum & $17(12)$ & 24 & 0.34 & 1.42 & 0.24 & 0.36 & 0.39 & 0.02 & 0.76 \\
\hline 58 & Dodonia viscose & $30(26)$ & 52 & 0.6 & 1.15 & 25.27 & 0.77 & 0.68 & 1.66 & 3.12 \\
\hline 59 & Echinochloa colona & $25(22)$ & 44 & 0.5 & 1.14 & 12.90 & 0.65 & 0.57 & 0.85 & 2.07 \\
\hline 60 & Eclipta prostrate & $17(14)$ & 28 & 0.34 & 1.21 & 9.77 & 0.42 & 0.39 & 0.64 & 1.45 \\
\hline 61 & Ecobolium viride & $18(15)$ & 30 & 0.36 & 1.20 & 6.45 & 0.45 & 0.41 & 0.42 & 1.28 \\
\hline 62 & Emila sonchifolia & $25(20)$ & 40 & 0.5 & 1.25 & 5.73 & 0.60 & 0.57 & 0.38 & 1.54 \\
\hline 63 & Euphorbia hirta & $16(13)$ & 26 & 0.32 & 1.23 & 6.52 & 0.39 & 0.36 & 0.43 & 1.18 \\
\hline 64 & Euphorbia serpens & $26(23)$ & 46 & 0.52 & 1.13 & 2.03 & 0.68 & 0.59 & 0.13 & 1.41 \\
\hline 65 & Euphorbia thymifolia & $29(17)$ & 34 & 0.58 & 1.71 & 7.80 & 0.51 & 0.66 & 0.51 & 1.68 \\
\hline 66 & Evolvulus alsinoides & $15(12)$ & 24 & 0.3 & 1.25 & 0.38 & 0.36 & 0.34 & 0.03 & 0.72 \\
\hline 67 & $\begin{array}{l}\text { Evolvulus } \\
\text { nummularis }\end{array}$ & $13(10)$ & 20 & 0.26 & 1.30 & 0.33 & 0.30 & 0.30 & 0.02 & 0.62 \\
\hline 68 & $\begin{array}{l}\text { Glycosmis } \\
\text { pentaphylla }\end{array}$ & $30(25)$ & 50 & 0.6 & 1.20 & 29.86 & 0.74 & 0.68 & 1.96 & 3.39 \\
\hline 69 & Gomphrena serata & $26(23)$ & 46 & 0.52 & 1.13 & 2.03 & 0.68 & 0.59 & 0.13 & 1.41 \\
\hline 70 & Gymnema sylvestre & $16(9)$ & 18 & 0.32 & 1.78 & 11.24 & 0.27 & 0.36 & 0.74 & 1.37 \\
\hline 71 & Hedyotis diffusa & $19(15)$ & 30 & 0.38 & 1.27 & 8.74 & 0.45 & 0.43 & 0.57 & 1.45 \\
\hline 72 & Hedyotis pterita & $14(10)$ & 20 & 0.28 & 1.40 & 5.71 & 0.30 & 0.32 & 0.37 & 0.99 \\
\hline 73 & $\begin{array}{l}\text { Helitrophihum } \\
\text { indicum }\end{array}$ & $29(23)$ & 46 & 0.58 & 1.26 & 24.43 & 0.68 & 0.66 & 1.60 & 2.95 \\
\hline 74 & Hemidesmus indicus & $19(14)$ & 28 & 0.38 & 1.36 & 3.66 & 0.42 & 0.43 & 0.24 & 1.09 \\
\hline 75 & Hibiscus micranthus & $13(9)$ & 18 & 0.26 & 1.44 & 5.98 & 0.27 & 0.30 & 0.39 & 0.96 \\
\hline 76 & Hibiscus surrattensis & $16(12)$ & 24 & 0.32 & 1.33 & 9.20 & 0.36 & 0.36 & 0.60 & 1.33 \\
\hline 77 & Hibiscus vitifolius & $15(12)$ & 24 & 0.3 & 1.25 & 4.04 & 0.36 & 0.34 & 0.26 & 0.96 \\
\hline 78 & $\begin{array}{l}\text { Hybanthus } \\
\text { ennaespermum }\end{array}$ & $13(10)$ & 20 & 0.26 & 1.30 & 1.68 & 0.30 & 0.30 & 0.11 & 0.70 \\
\hline 79 & $\begin{array}{l}\text { Hygrophila } \\
\text { auriculata }\end{array}$ & $28(25)$ & 50 & 0.56 & 1.12 & 6.42 & 0.74 & 0.64 & 0.42 & 1.80 \\
\hline 80 & Hyptis suvaledens & $25(17)$ & 34 & 0.5 & 1.47 & 14.37 & 0.51 & 0.57 & 0.94 & 2.02 \\
\hline 81 & Imperata cylindrical & $29(18)$ & 36 & 0.58 & 1.61 & 24.43 & 0.54 & 0.66 & 1.60 & 2.80 \\
\hline 82 & $\begin{array}{l}\text { Indigofera } \\
\text { asplanthoides }\end{array}$ & $32(27)$ & 54 & 0.64 & 1.19 & 1.83 & 0.80 & 0.73 & 0.12 & 1.65 \\
\hline 83 & Indigofera glandulosa & $20(9)$ & 18 & 0.4 & 2.22 & 1.15 & 0.27 & 0.46 & 0.08 & 0.80 \\
\hline
\end{tabular}




\begin{tabular}{|c|c|c|c|c|c|c|c|c|c|c|}
\hline 84 & Indigofera hirsute & $29(25)$ & 50 & 0.58 & 1.16 & 0.74 & 0.74 & 0.66 & 0.05 & 1.45 \\
\hline 85 & Indigofera uniflora & $29(17)$ & 34 & 0.58 & 1.71 & 0.42 & 0.51 & 0.66 & 0.03 & 1.19 \\
\hline 86 & Ipomea carnea & $13(8)$ & 16 & 0.26 & 1.63 & 1.32 & 0.24 & 0.30 & 0.09 & 0.62 \\
\hline 87 & Ipomea obscura & $22(16)$ & 32 & 0.44 & 1.38 & 1.72 & 0.48 & 0.50 & 0.11 & 1.09 \\
\hline 88 & Justicia adothoda & $12(8)$ & 16 & 0.24 & 1.50 & 12.92 & 0.24 & 0.27 & 0.85 & 1.36 \\
\hline 89 & Justicia gluca & $32(26)$ & 52 & 0.64 & 1.23 & 6.17 & 0.77 & 0.73 & 0.40 & 1.91 \\
\hline 90 & Justicia simplex & $18(13)$ & 26 & 0.36 & 1.38 & 1.03 & 0.39 & 0.41 & 0.07 & 0.87 \\
\hline 91 & $\begin{array}{l}\text { Justicia } \\
\text { tranquebariensis }\end{array}$ & $26(20)$ & 40 & 0.52 & 1.30 & 1.49 & 0.60 & 0.59 & 0.10 & 1.29 \\
\hline 92 & Kyllinga odorata & $29(19)$ & 38 & 0.58 & 1.53 & 0.74 & 0.57 & 0.66 & 0.05 & 1.28 \\
\hline 93 & Lagascea mollis & $26(20)$ & 40 & 0.52 & 1.30 & 2.65 & 0.60 & 0.59 & 0.17 & 1.36 \\
\hline 94 & Lanata camera & $20(15)$ & 30 & 0.4 & 1.33 & 19.90 & 0.45 & 0.46 & 1.31 & 2.21 \\
\hline 95 & Leucas aspera & $55(50)$ & 100 & 1.1 & 1.10 & 14.80 & 1.49 & 1.25 & 0.97 & 3.71 \\
\hline 96 & Leucasgrandis & $19(14)$ & 28 & 0.38 & 1.36 & 7.75 & 0.42 & 0.43 & 0.51 & 1.36 \\
\hline 97 & Linderina ciliate & $19(15)$ & 30 & 0.38 & 1.27 & 3.66 & 0.45 & 0.43 & 0.24 & 1.12 \\
\hline 98 & Linderina crustacea & $26(20)$ & 40 & 0.52 & 1.30 & 3.35 & 0.60 & 0.59 & 0.22 & 1.41 \\
\hline 99 & Ludwigia octovalis & $14(12)$ & 24 & 0.28 & 1.17 & 1.81 & 0.36 & 0.32 & 0.12 & 0.79 \\
\hline 100 & Ludwigia peruviana & $16(10)$ & 20 & 0.32 & 1.60 & 2.06 & 0.30 & 0.36 & 0.14 & 0.80 \\
\hline 101 & $\begin{array}{l}\text { Malvastrum } \\
\text { coromandelianum }\end{array}$ & $17(12)$ & 24 & 0.34 & 1.42 & 6.09 & 0.36 & 0.39 & 0.40 & 1.14 \\
\hline 102 & Martynia annua & $39(30)$ & 60 & 0.78 & 1.30 & 38.81 & 0.89 & 0.89 & 2.55 & 4.33 \\
\hline 103 & $\begin{array}{l}\text { Melhania } \\
\text { hamiltoniana }\end{array}$ & $29(25)$ & 50 & 0.58 & 1.16 & 0.42 & 0.74 & 0.66 & 0.03 & 1.43 \\
\hline 104 & $\begin{array}{l}\text { Melochia } \\
\text { corehorifolia }\end{array}$ & $45(36)$ & 72 & 0.9 & 1.25 & 12.11 & 1.07 & 1.03 & 0.79 & 2.89 \\
\hline 105 & Merremia tridentate & $30(26)$ & 52 & 0.6 & 1.15 & 1.72 & 0.77 & 0.68 & 0.11 & 1.57 \\
\hline 106 & $\begin{array}{l}\text { Microstachys } \\
\text { chamaelea }\end{array}$ & $17(12)$ & 24 & 0.34 & 1.42 & 1.33 & 0.36 & 0.39 & 0.09 & 0.83 \\
\hline 107 & Mimosa pudica & $27(19)$ & 38 & 0.54 & 1.42 & 6.19 & 0.57 & 0.62 & 0.41 & 1.59 \\
\hline 108 & Mollugo pentaphylla & $50(38)$ & 76 & 1 & 1.32 & 3.90 & 1.13 & 1.14 & 0.26 & 2.53 \\
\hline 109 & Naregama alata & $20(16)$ & 32 & 0.4 & 1.25 & 6.24 & 0.48 & 0.46 & 0.41 & 1.34 \\
\hline 110 & Ocimum americanum & $19(14)$ & 28 & 0.38 & 1.36 & 13.34 & 0.42 & 0.43 & 0.88 & 1.73 \\
\hline 111 & Ocimum basilicum & $20(15)$ & 30 & 0.4 & 1.33 & 19.90 & 0.45 & 0.46 & 1.31 & 2.21 \\
\hline 112 & Ocimum gratissimum & $22(14)$ & 28 & 0.44 & 1.57 & 15.45 & 0.42 & 0.50 & 1.01 & 1.93 \\
\hline 113 & Ocimum tenuiflorum & $25(20)$ & 40 & 0.5 & 1.25 & 6.73 & 0.60 & 0.57 & 0.44 & 1.61 \\
\hline 114 & $\begin{array}{l}\text { Oldenlandia } \\
\text { corymbosa }\end{array}$ & $23(21)$ & 42 & 0.46 & 1.10 & 0.33 & 0.63 & 0.52 & 0.02 & 1.17 \\
\hline
\end{tabular}




\begin{tabular}{|c|c|c|c|c|c|c|c|c|c|c|}
\hline 115 & $\begin{array}{l}\text { Oldenlandia } \\
\text { umbellate }\end{array}$ & $65(46)$ & 92 & 1.3 & 1.41 & 6.62 & 1.37 & 1.48 & 0.43 & 3.29 \\
\hline 116 & $\begin{array}{l}\text { Orthosiphon } \\
\text { thymiflorus }\end{array}$ & $23(19)$ & 38 & 0.46 & 1.21 & 19.37 & 0.57 & 0.52 & 1.27 & 2.36 \\
\hline 117 & Osbeckia aspera & $39(35)$ & 70 & 0.78 & 1.11 & 10.50 & 1.04 & 0.89 & 0.69 & 2.62 \\
\hline 118 & Pavetta indica & $46(28)$ & 56 & 0.92 & 1.64 & 42.19 & 0.83 & 1.05 & 2.77 & 4.65 \\
\hline 119 & Pedalium тихех & $45(35)$ & 70 & 0.9 & 1.29 & 18.34 & 1.04 & 1.03 & 1.20 & 3.27 \\
\hline 120 & Pergularia daemia & $30(26)$ & 52 & 0.6 & 1.15 & 6.88 & 0.77 & 0.68 & 0.45 & 1.91 \\
\hline 121 & $\begin{array}{l}\text { Peristrophe } \\
\text { paniculata }\end{array}$ & $32(29)$ & 58 & 0.64 & 1.10 & 1.83 & 0.86 & 0.73 & 0.12 & 1.71 \\
\hline 122 & Persicaria hydropiper & $29(23)$ & 46 & 0.58 & 1.26 & 5.59 & 0.68 & 0.66 & 0.37 & 1.71 \\
\hline 123 & Phryma laptostachya & $23(19)$ & 38 & 0.46 & 1.21 & 1.79 & 0.57 & 0.52 & 0.12 & 1.21 \\
\hline 124 & Phyla nodiflora & $15(12)$ & 24 & 0.3 & 1.25 & 1.17 & 0.36 & 0.34 & 0.08 & 0.78 \\
\hline 125 & Phyllanthus amarus & $60(50)$ & 100 & 1.2 & 1.20 & 7.74 & 1.49 & 1.37 & 0.51 & 3.36 \\
\hline 126 & Phyllanthus debilis & $25(23)$ & 46 & 0.5 & 1.09 & 3.22 & 0.68 & 0.57 & 0.21 & 1.47 \\
\hline 127 & $\begin{array}{l}\text { Phyllanthus } \\
\text { maderraptensis }\end{array}$ & $23(20)$ & 40 & 0.46 & 1.15 & 2.34 & 0.60 & 0.52 & 0.15 & 1.27 \\
\hline 128 & $\begin{array}{l}\text { Phyllanthus } \\
\text { myrtifolius }\end{array}$ & $39(31)$ & 62 & 0.78 & 1.26 & 3.97 & 0.92 & 0.89 & 0.26 & 2.07 \\
\hline 129 & Phyllanthus virgatus & $28(21)$ & 42 & 0.56 & 1.33 & 1.11 & 0.63 & 0.64 & 0.07 & 1.34 \\
\hline 130 & Physalis minima & $30(25)$ & 50 & 0.6 & 1.20 & 3.06 & 0.74 & 0.68 & 0.20 & 1.63 \\
\hline 131 & Plumbago zeylanica & $19(15)$ & 30 & 0.38 & 1.27 & 2.45 & 0.45 & 0.43 & 0.16 & 1.04 \\
\hline 132 & $\begin{array}{l}\text { Polycarpaea } \\
\text { corrymbosa }\end{array}$ & $32(29)$ & 58 & 0.64 & 1.10 & 7.34 & 0.86 & 0.73 & 0.48 & 2.07 \\
\hline 133 & Polygala chinensis & $20(16)$ & 32 & 0.4 & 1.25 & 1.15 & 0.48 & 0.46 & 0.08 & 1.01 \\
\hline 134 & Portulaca quadrifida & $29(26)$ & 52 & 0.58 & 1.12 & 24.43 & 0.77 & 0.66 & 1.60 & 3.04 \\
\hline 135 & Psuedathria viscid & $35(27)$ & 54 & 0.7 & 1.30 & 3.57 & 0.80 & 0.80 & 0.23 & 1.84 \\
\hline 136 & Rauvolfia serpentine & $9(7)$ & 14 & 0.18 & 1.29 & 2.06 & 0.21 & 0.21 & 0.14 & 0.55 \\
\hline 137 & $\begin{array}{l}\text { Rhinacanthus } \\
\text { nasutus }\end{array}$ & $20(15)$ & 30 & 0.4 & 1.33 & 16.85 & 0.45 & 0.46 & 1.11 & 2.01 \\
\hline 138 & Rhynchosia minima & $35(30)$ & 60 & 0.7 & 1.17 & 24.58 & 0.89 & 0.80 & 1.61 & 3.30 \\
\hline 139 & Rulliea prostrate & $35(29)$ & 58 & 0.7 & 1.21 & 16.11 & 0.86 & 0.80 & 1.06 & 2.72 \\
\hline 140 & Rulliea tuberose & $19(17)$ & 34 & 0.38 & 1.12 & 10.92 & 0.51 & 0.43 & 0.72 & 1.66 \\
\hline 141 & Rungia repens & $15(11)$ & 22 & 0.3 & 1.36 & 2.89 & 0.33 & 0.34 & 0.19 & 0.86 \\
\hline 142 & Scoparia dulcis & $29(20)$ & 40 & 0.58 & 1.45 & 20.36 & 0.60 & 0.66 & 1.34 & 2.59 \\
\hline 143 & Senna uniflora & $12(10)$ & 32 & 0.4 & 1.25 & 10.32 & 0.48 & 0.46 & 0.68 & 1.61 \\
\hline 144 & Senna oxidentalis & $20(16)$ & 20 & 0.24 & 1.20 & 4.30 & 0.30 & 0.27 & 0.28 & 0.85 \\
\hline
\end{tabular}




\begin{tabular}{|c|c|c|c|c|c|c|c|c|c|c|}
\hline 145 & Sida acuta & $15(10)$ & 20 & 0.3 & 1.50 & 1.93 & 0.30 & 0.34 & 0.13 & 0.77 \\
\hline 146 & Sida cardata & $59(50)$ & 100 & 1.18 & 1.18 & 2.35 & 1.49 & 1.35 & 0.15 & 2.99 \\
\hline 147 & Sida cordifolia & $15(12)$ & 24 & 0.3 & 1.25 & 12.64 & 0.36 & 0.34 & 0.83 & 1.53 \\
\hline 148 & Solanum melongena & $16(10)$ & 20 & 0.32 & 1.60 & 5.73 & 0.30 & 0.36 & 0.38 & 1.04 \\
\hline 149 & Solanum torvum & $20(13)$ & 26 & 0.4 & 1.54 & 11.50 & 0.39 & 0.46 & 0.75 & 1.60 \\
\hline 150 & Solanum virginianum & $25(19)$ & 38 & 0.5 & 1.32 & 11.50 & 0.57 & 0.57 & 0.76 & 1.89 \\
\hline 151 & $\begin{array}{l}\text { Spermacoce } \\
\text { articularis }\end{array}$ & $17(13)$ & 26 & 0.34 & 1.31 & 1.73 & 0.39 & 0.39 & 0.11 & 0.89 \\
\hline 152 & Spermacoce hispida & $22(19)$ & 50 & 0.64 & 1.28 & 3.26 & 0.74 & 0.73 & 0.21 & 1.69 \\
\hline 153 & $\begin{array}{l}\text { Spermacoce } \\
\text { ocymoides }\end{array}$ & $15(10)$ & 38 & 0.44 & 1.16 & 2.84 & 0.57 & 0.50 & 0.19 & 1.25 \\
\hline 154 & Spermacoce pusilla & $32(25)$ & 20 & 0.3 & 1.50 & 1.93 & 0.30 & 0.34 & 0.13 & 0.77 \\
\hline 155 & $\begin{array}{l}\text { Stashytropheta } \\
\text { jamensia }\end{array}$ & $29(19)$ & 38 & 0.58 & 1.53 & 26.60 & 0.57 & 0.66 & 1.75 & 2.97 \\
\hline 156 & $\begin{array}{l}\text { Stylosanthus } \\
\text { fruticona }\end{array}$ & $19(15)$ & 30 & 0.38 & 1.27 & 2.45 & 0.45 & 0.43 & 0.16 & 1.04 \\
\hline 157 & Stylosantus hamate & $46(39)$ & 78 & 0.92 & 1.18 & 4.69 & 1.16 & 1.05 & 0.31 & 2.52 \\
\hline 158 & Syndrella nodiflora & $19(13)$ & 26 & 0.38 & 1.46 & 5.11 & 0.39 & 0.43 & 0.34 & 1.16 \\
\hline 159 & Tephrosia purpurea & $77(48)$ & 96 & 1.54 & 1.60 & 82.89 & 1.43 & 1.76 & 5.44 & 8.62 \\
\hline 160 & $\begin{array}{l}\text { Trianthema } \\
\text { portulacastrum }\end{array}$ & $29(22)$ & 44 & 0.58 & 1.32 & 16.67 & 0.65 & 0.66 & 1.09 & 2.41 \\
\hline 161 & Tribulus terrestrus & $29(19)$ & 38 & 0.58 & 1.53 & 7.80 & 0.57 & 0.66 & 0.51 & 1.74 \\
\hline 162 & $\begin{array}{l}\text { Trichodesmium } \\
\text { indicum }\end{array}$ & $35(29)$ & 58 & 0.7 & 1.21 & 18.06 & 0.86 & 0.80 & 1.19 & 2.85 \\
\hline 163 & Tridax procumbens & $30(27)$ & 54 & 0.6 & 1.11 & 23.12 & 0.80 & 0.68 & 1.52 & 3.01 \\
\hline 164 & $\begin{array}{l}\text { Triumfetta } \\
\text { rhomboids }\end{array}$ & $29(21)$ & 42 & 0.58 & 1.38 & 14.96 & 0.63 & 0.66 & 0.98 & 2.27 \\
\hline 165 & Urena lobata & $29(23)$ & 46 & 0.58 & 1.26 & 7.80 & 0.68 & 0.66 & 0.51 & 1.86 \\
\hline 166 & Urena sinuate & $27(22)$ & 44 & 0.54 & 1.23 & 11.01 & 0.65 & 0.62 & 0.72 & 1.99 \\
\hline 167 & Vernonia cineria & $35(27)$ & 54 & 0.7 & 1.30 & 8.03 & 0.80 & 0.80 & 0.53 & 2.13 \\
\hline 168 & Vigna trilobata & $30(26)$ & 52 & 0.6 & 1.15 & 9.36 & 0.77 & 0.68 & 0.61 & 2.07 \\
\hline 169 & Waltheria indica & $25(18)$ & 36 & 0.5 & 1.39 & 10.19 & 0.54 & 0.57 & 0.67 & 1.77 \\
\hline 170 & Xanthium stumarium & $26(21)$ & 42 & 0.52 & 1.24 & 21.90 & 0.63 & 0.59 & 1.44 & 2.66 \\
\hline \multirow[t]{2}{*}{171} & Zorina diphylla & $20(15)$ & 30 & 0.4 & 1.33 & 4.59 & 0.45 & 0.46 & 0.30 & 1.20 \\
\hline & Total & & 6720 & 87.68 & 227.34 & 1523.65 & 100.00 & 100.00 & 100.00 & 300.00 \\
\hline
\end{tabular}


The higher IVI contributed by many species like Tephrosia purpurea, Clerodendron infortunatum Acalypa indica, Aerva lanata, Asystasia gangetica, Belpharis maderaptensis, Cleome viscosa, Dodonia viscose, Glycosmis pentaphylla, Leucas aspera, Limnophila indica, Martynia annua, Oldenlandia umbellate, Pavetta indica, Phyllanthus amarus and Rhynchosia minima in the present study area indicates their stronger perpetuation and higher functional role in the ecosystem.

Many species like Alteranthera pungens, Amaranthus spinosus, Desmodium illinoensis, Evolvulus nummularis, Hybanthus enneaspermus, Justicia simplex, Ocimum basilicum, Plumbago zeylanica, Phyla nodiflora, Rauvolfia serpentine, Rungia repens, Solanum melongena, Acanthospermum hispidum, Aerva javanica, Amaranthus viridis, A. spinosus, Andrographis paniculata, Asparagus racemosus, Barleria buxifolia, B.lupulina, B. mysorensis, Biophytum sensitivum, Blainvilla trinervia, Borreria articularis, Boerhavia diffusa, Senna occidentalis, Cardiospermum halicabum, Centella asiatica, Chromolaena oderata, Cleome aspera, C. tetrandra, C. rutidesperma, Clitoria ternate, Commelina benghalensis, Corchorus aestuans, Croton hirtus, C. bonplandiam, C. sparsiflorus, Curculigo orchioides, Cyanotis axillaris, Cyanotis tuberosa, Desmodium adscendens, Desmodium triflorum, Evolvulus alsinoides, Ecobolium viride, Euphorbia hirta, Heydyotis peterita, Hibiscus vitifolius, $H$. micranthus, Indigofera glandulosa, Ipomea carnea, Justicia gluca, Ludwigia octovalvis, L. Peruviana, Phyllanthus virgatus, Sebastiana chamaelea, Sida acuta, Spermacoce articularis and S. hispida were weaker in ecological attributes and secured poor IVI in the communities of studied forest. In addition to less fitness, the killing of young individuals by frost in winter may be ascribed as reason for this fact (Agarwal et al., 1961). Hence it is suggested that priority must be given to these species for conservation by employing proper macro micro propagation techniques.

\section{REFERENCES}

Agarwal, S.C., U.S. Madan, S. Chinnamani and N.D.Rege, (1961). Ecological studies in the Nilgiris. Indian Forester 87(6): 376-389.

Ahmedullah, M. and M.P. Nayar, (1987). Endemic Plants of the Indian Region. Vol.1. Botanical Survey of India, Howrah.

Anonymous, (1940-1976). The wealth of India; $A$ dictionary of Indian Raw Materials and
Industrial Products Raw Materials. Vols. 1-11/

C.S.I.R., New Delhi.

Belsky, A.J. (1988). Regional influence on small scale vegetational heterogeneity within grasslands in the Serengeti National Park, Tanzania, Vegetatio 74: 7-10.

Champion, H.G. and S.K. Seth. (1968). A revised survey of the forest types of India. Government of India Press. Nasik, India. pp. 404.

Chandrasekaran, S. and P.S. Swamy, (1995). Changes in the herbaceous vegetation following disturbance due to biotic interference in natural and man-made ecosystems, Western Ghats. Tropical Ecology. 36: 213-220.

Cottam, G. and J.T. Curtis, (1956). The use of distance measures in phytosociological sampling. Ecology $37: 451: 460$.

Gadgil, M. (1984). An approach to ecodevelopment of Western Ghats. The Administrator 29(4) : 339379.

Garkoti, S.C. and S.P. Singh, (1997). Structure and function of herbaceous vegetation in high mountains of central Himalaya. Tropical Ecology 38(1):153-156.

Maheshwari, J.K. (2000). Ethnobiology and Medicinal Plants of Indian Subcontinent. Scientific Publications, Jodhpur.

Nayar, M.P. and Sastry, A.R.K. (1987-1990). Red Data Book of Indian Plants. Vols. 1-3: Botanical Survey of India, Howrah.

Padmavathy, S. (2005). Ecological investigations for the identification of plants of conservation importance in the understories of certain shola forests at Manjur, the Nilgiris, Western Ghats, India. Ph.D., thesis, Bharathiar University, Coimbatore. India.

Puri, G.S., Gupta, R.K. and Meher-Homji, V.M. (1989). Temperate forests of India including tropical montane forests. In: G.S. Puri (ed.) Forest Ecology Vol. 2:49-55.

Saxena, K.G. (1991). Biological invasions in the Indian subcontinent: Review of invasion by plants. In: P.S.Ramakrishnan (ed.). Ecology of biological invasion in the tropics. International Scientific Publications, New Delhi.

Singh, S.K. (2004). Ethnomedicinal plants of Kulluvally, Himachalpradesh. Journal of Non Timber Forest Products 11(1):74-79.

Usher, M.B. (1991). Biological invasions into tropical nature. In: Ecology of Biological invasion in the Tropics. (Ed.) Ramakrishonan, P.S. International Scientific Publications, New Delhi. p. 21-34. 